\title{
Kommentar zu Lindner M. A. et al. (2021). Ein Plädoyer zur Qualitätssicherung schriftlicher Prüfungen im Psychologiestudium
}

\author{
Winfried Rief
}

Philipps-Universität Marburg

Das Positionspapier enthält zahlreiche hilfreiche und wichtige Hinweise, die bei der Konstruktion schriftlicher Prüfungen zu beachten sind. Damit trägt es zur kontinuierlichen Qualitätsverbesserung dieser Prüfungen bei. Auch die im Positionspapier mehrfach erwähnte Notwendigkeit, für die Studierenden hier transparente und berechenbare Algorithmen vorzugeben, kann nur ausnahmslos unterstützt werden.

Neben diesen Stärken gibt es aus klinischer Sicht, jedoch auch aus allgemeiner psychologischer Perspektive einige Aspekte, die einer weiteren Diskussion Wert sind. Durch das neue Psychotherapeutengesetz werden neue Prüfungsformate eingeführt und erhalten zentralen Stellenwert, auf die jedoch in dem vorliegenden Positionspapier nicht eingegangen werden konnte. Praktische, handlungsorientierte Kompetenzen sollen mit Schauspielpatient_innen geprüft werden. Insofern warten die klinischinteressierten Kolleginnen und Kollegen auf das Fortsetzungsmanuskript, das gerade auf die Qualitätsmerkmale und Verbesserungsmöglichkeiten solcher handlungsorientierter Prüfungen einschließlich ihrer zahlreichen, damit einhergehenden Probleme eingeht.

Ein weiteres Dilemma, das noch mehr Diskussion verdient, ist die in der Regel konfligierende Zielsetzung von Prüfungen: Einerseits sollen sie einen möglichst direkten Bezug zu Lehrinhalten haben, sozusagen einen Dialog zwischen Lehre und Prüfung ermöglichen, sehr modern dann auch unter Hinzuziehung der Studierenden selbst, andererseits wird oftmals der Wunsch mit Prüfungen verbunden, eine Vergleichbarkeit der Leistungsniveaus von Absolvierenden unterschiedlicher Universitäten und Bundesländer herzustellen. Je nach Funktion der Prüfung kann meines Erachtens das eine oder andere mehr gewichtet werden. Die Vergleichbarkeit der Abschlüsse zwischen verschiedenen Universitäten ist z. B. ein zwar unter manchen Perspektiven relevantes Ziel, oftmals wird jedoch noch wichtiger sein, dass der Lernerfolg aus der Lehre möglichst tief und langanhaltend ist. Für dieses letztgenannte Ziel scheint die lehrveranstaltungsspezifische $\mathrm{Ab}$ stimmung und gegenseitige Bereicherung von Lehre versus Prüfungsformat und Prüfungsinhalt wichtiger zu sein.

In moderner Zeit fordern wir grundsätzlich, dass Prüfungen nicht einfach Wissen abfragen, sondern Ausbil- dungsziele und erworbene Kompetenzen abtesten sollen. Während das Positionspapier stark auf den Prüfungsteil dieser Komponenten eingeht, erscheint mir ein Blick auch auf die Definition von Ausbildungszielen und Kompetenzen unerlässlich, um unsere aktuellen Prüfungen zu diskutieren. Könnte es nicht sein, dass wir oftmals zu oberflächlich Ausbildungsziele und Zielkompetenzen definieren, diese dann aber methodisch hoch anspruchsvoll prüfen? Aus meiner akademischen Erfahrung würde ich behaupten, dass die früher rein wissensbezogene Definition von Ausbildungszielen oftmals etwas lieblos in kompetenzbasierte Ziele übertragen wurde. Wenn aber bereits an der Stelle der Definition der zu prüfenden Kompetenzmerkmale ein substantieller Qualitätsmangel ist, wird sich dieser zwangsläufig auch auf den Sinn und die Qualität der Prüfungsergebnisse niederschlagen.

Zieht man nochmals die klinische Perspektive der Approbationsprüfung heran, so ist gerade da die praktische Kompetenzbasierung von enorm hohem Stellenwert. Zusätzlich kommt die Aufgabe der Selektion durch die Prüfung hinzu, in einer Härte, wie wir sie sonst vielleicht nicht kennen. Die Approbationsprüfung soll sicherstellen, dass nur diese Personen mit der nötigen fachlichen und menschlichen Kompetenz in der Heilkunde tätig werden, die verantwortlich zum Wohle der Patientinnen und Patienten handeln und Leid, Negativeffekte, Gefahren von ihnen abwenden. Damit geht es in der Approbationsprüfung eigentlich auch darum, Personen auszuschließen, die später nicht in der Heilkunde mit bedürftigen Personen tätig werden sollen. Man wird schnell darüber Einigkeit herstellen, dass dies nicht ausschließlich durch Prüfung von Wissenskompetenz entschieden werden kann. Aber es fehlt bislang noch an leicht objektivierbaren anderen Kriterien, um diese gesellschaftlich wichtige Entscheidung zu treffen. Manche Prüferinnen und Prüfer werden an manchen Stellen ein stark subjektives Gefühl haben, dass ein bestimmter Prüfling nicht heilkundlich tätig werden sollte - und dies aber schlecht durch justiziable Prüfungsleistungen begründen können. Hier wünscht sich die klinische Psychologie noch mehr Unterstützung von unseren Expertinnen und Experten aus dem Bereich Diagnostik, wie mit solchen gesellschaftlich hoch relevanten Entscheidungen verfahren werden kann. 
Last not least nochmals ein positiver Kommentar zum Positionspapier von Lindner et al. (2021): Von besonderer Relevanz fand ich den Hinweis, dass wir noch mehr und kreativer die Möglichkeiten der modernen Technik nutzen können und müssen. Ein kurzer Videoclip von einer Schauspielpatientin bzw. einem Schauspielpatienten, zu der/dem anschließend Fragen gestellt werden; ein Videoclip zum Therapeut_innenverhalten, das anschließend bewertet werden muss; standardisierte Internet-Dialoge zwischen Schauspiel-Patient_innen und Prüfenden sowie Prüfungskommission; sich nach bestimmten Algorithmen weiterentwickelnde Prüfungssequenzen: Hier erscheinen die Möglichkeiten extrem vielfältig und für eine bessere Kompetenz-Prüfung sehr zielführend, jedoch bislang großteils ungenutzt.

Die in der akademischen Psychologie vorhandene diagnostische Kompetenz hat viel zu bieten; ein intensiver subdisziplinärer Dialog mit anderen psychologischen Be- reichen kann wesentliches für die Herausforderungen der Gesellschaft liefern.

\section{Literatur}

Lindner, M. A., Sparfeldt, J. R., Köller, O., Lukas, J \& Leutner, D. (2021). Ein Plädoyer zur Qualitätssicherung schriftlicher Prüfungen im Psychologiestudium. Psychologische Rundschau, 72, 93 -105. https://doi.org/10.1026/0033-3042/a000524

\section{Prof. Dr. Winfried Rief}

Philipps-Universität Marburg

Biegenstraße 10

35037 Marburg

rief@staff.uni-marburg.de

https://doi.org/10.1026/0033-3042/a000534 